\title{
Association of Serum Ferritin Concentration and Lung Function in the Tobacco-naiive Population: Retrospective population-based study of KNHANES V
}

\section{Taeyun Kim}

Kosin University Gospel Hospital https://orcid.org/0000-0001-7786-5051

\section{Hyunji Choi}

Kosin University Gospel Hospital

Jihun Kang ( $\nabla$ josua85@naver.com )

https://orcid.org/0000-0002-2263-9054

\section{Research article}

Keywords: Ferritin, smoking, Lung function, Tobacco-naïve, KNHANES

Posted Date: November 6th, 2019

DOl: https://doi.org/10.21203/rs.2.16883/v1

License: (c) (1) This work is licensed under a Creative Commons Attribution 4.0 International License.

Read Full License 


\section{Abstract}

Background: Ferritin regulates iron homeostasis, and is involved in the inflammation in the lung, especially in smokers; however, its effects on pulmonary function in non-smokers remain unclear. The present study aimed to evaluate the association between serum ferritin and lung function in a tobacconaïve population. Methods: In this study, 25,534 individuals were enrolled, among who 5,338 tobacconaïve individuals were identified; of those, 342 men and 2,879 women (742 pre- and 2,137 postmenopausal) with data of serum ferritin, lung function, and covariates were included. To evaluate the association of ferritin and lung function, multivariable-adjusted linear regression analyses was used including the factors of predicted value of forced expiratory volume in 1 second (FEV $1 \%$ ) and forced vital capacity (FVC\%) . Logistic regression analyses were used to measure the relationship between ferritin and restrictive and obstructive lung disease. Results: In pre-menopausal women, FEV 1 \%/FVC was weakly but positively associated with serum ferritin, and after adjusting for covariates, the association was without statistical significance. No significant association between ferritin and obstructive lung disease was observed. In post-menopausal women, predicted FVC\% was negatively associated with serum ferritin, and ferritin was dose-dependently related with risk for restrictive lung disease. The odds ratio for restrictive lung disease in post-menopausal women was 2.285 at Q3 and 1.560 at Q2 relative to that at Q1. Conclusions: High serum ferritin level was significantly associated with lower $\mathrm{FVC} \%$ and increased risk of restrictive lung disease in tobacco-naïve post-menopausal women. Further study is needed to determine the mechanism underlying the current findings.

\section{Background}

Ferritin is an important modulating factor of the body's iron homeostasis (1). Although ferritin is a ubiquitous regulator of in vivo iron metabolism, many reports have indicated that ferritin contributed to oxidative tissue damage by producing free radicals (2-4). The ferritin level is considered to reflect acute and chronic inflammatory processes $(5,6)$, and based on this fact, elevated ferritin level may be a reliable inflammatory biomarker.

Tobacco contains a significant amount of iron (440-1150 $\mu \mathrm{g} / \mathrm{g})$, and hence, tobacco smoking can affect both the alveolar and systemic ferritin concentration $(7,8)$. Elevation of the ferritin level of the pulmonary tissue could cause airway inflammation $(9,10)$. Nelson and colleagues reported that the extracellular ferritin concentration through broncho-alveolar lavage test was higher in smokers than nonsmokers, which suggests that high ferritin may be related to emphysema and lung cancer (11). Few previous studies estimated a role of ferritin in lung function, however the effects of ferritin on pulmonary function in the non-smoking population is unclear.

Epidemiologic studies evaluating the effect of ferritin on lung function reported conflicting results (12, 13). Previous studies conducted analyses with adjustment for smoking-related factors such as pack-year and smoking status; nevertheless, the effects of smoking outweigh other covariates, and studies excluding the smoking population are needed for objective evaluation of the influence of ferritin. 
Moreover, no study on the association between ferritin and lung function in a tobacco-naïve population excluding both current and second-hand smokers, is reported in the literature.

In the present study, we aimed to evaluate the relationship between serum ferritin concentration and lung function in the tobacco-naïve population using a nationally representative sample.

\section{Methods}

\section{Study subjects}

Korea National Health and Nutritional Examination Survey (KNHANES) is a nationally representative cross-sectional survey of non-institutionalized South Korean citizens which is conducted annually by the Korean Center for Disease Control and Prevention (KCDC). Details of the study design and protocol are described in a previous report (14). The survey comprises three main components of a health interview, health examination, and nutritional survey, and the data of socioeconomic status, demographic characteristics, health behaviors, quality of life, biochemical data, and dietary intake are examined. KNHANES is designed as a complex sample survey, and hence, nationally representative data are obtained by stratified multistage clustered probability sampling; complete raw data are freely available to the public on the KCDC website.

In the present study, data of KNHANES $\otimes$ conducted from 2010 to 2012 were used, which are the first years with data of all iron indices available, including the serum ferritin level, serum iron level, total iron binding capacity (TIBC), and daily iron intake; the ferritin concentration was examined only for the period of 2007 to 2012. Of 31,596 eligible individuals, 25,534 individuals participated in KNHANES 2010-2012, with response rate of $80.8 \%$. Pulmonary function test (PFT) was performed in 9,281 participants aged over 40 years (4,024 men and 5,257 women). First, 540 men and 3,432 women were identified with both non-smoking and no second-hand smoking. Subsequently, subjects with clinical conditions that may affect serum ferritin level were excluded as follows: Active malignancy $(n=196)$, treatment for tuberculosis $(n=5)$, suspicious findings of active tuberculosis or pneumonia on chest radiograph $(n=$ $12)$, and hepatitis $B$ or $C(n=70)$, with overlap of subjects in any combination of two categories. In addition, subjects with missing data for variables of interest were excluded. Finally, 342 men and 2,879 women (742 pre-menopausal and 2,137 post-menopausal women) were included in the analysis.

The study protocol was approved by the Institutional Review Board (IRB) of the KCDC (IRB No. 201002CON-21-C in 2010, 2011-02CON-06-C in 2011, and 2012-01EXP-01-2C in 2012), and the study was conducted according to the tenets of the Declaration of Helsinki. Moreover, all study procedures were in accordance with STROBE guidelines, and written informed consent was obtained from all individuals before their participation in KNHANES.

\section{Data collection}


Smoking status was categorized into active smokers, passive smokers, and non-smokers based on WHO classification. Non-smokers were defined as subjects who reported smoking $<100$ cigarettes during their lifetime, and no smoking at the time of survey. Since even self-reported non-smokers may be exposed to second-hand smoking in the workplace or home, the participants with second-hand smoking were confirmed based on their response to the question: Have you ever been exposed to second-hand smoking in work place or at home? Tobacco-naïve population was defined as those who are non-smokers without exposure to second-hand smoking both at work and home.

PFT was performed only in subjects aged $\geq 40$ years by trained medical technicians using Dry rolling seal spirometer (Model 2130, SensorMedics; Yorba Linda, CA, USA). Standardization of spirometry was done based on the criteria of American Thoracic Society and European Respiratory Society (15). Obstructive lung disease (OLD) was defined as the ratio of forced expiratory volume in 1 second $\left(\mathrm{FEV}_{1}\right)$ divided by forced vital capacity (FVC) of $<70 \%$ based on the Global Initiative for Chronic Obstructive Lung Disease (GOLD) classification system (16). Restrictive lung disease (RLD) was defined as $F E V_{1} / F V C$ of $\geq 70 \%$ and FVC of $<80 \%$.

Information of the subjects' socio-economic characteristics (age, residential area, education, household income) was collected during face-to-face interviews. Residential area was categorized into two groups (rural or urban); education was categorized into three groups (middle school or lower, high school, and college or more); household income level was categorized into four groups (low, lower middle, upper middle, and high), and calculated by dividing the mean monthly income by the square root of the number of family members. Data of hormonal therapy (HRT) use, including oral HRT or oral contraceptives for at least 1 month was assessed through self-reporting questionnaire.

In all subjects, the laboratory samples were obtained by skilled medical assistants and transported to the Central Laboratory (NEODIN Medical Institute, Seoul, South Korea) that met the quality-control standards of an extramural institute (Hallym University, Seoul, South Korea). Hemoglobin level $(\mathrm{g} / \mathrm{dL})$ was measured with XE-2100D (Sysmex; Japan) using cyanide-free method. Serum ferritin level ( $\mathrm{ng} / \mathrm{mL}$ ) was measured with 1470 WIZARD gamma-Counter (PerkinElmer; Finland) using immuno-radiometric assay. Serum iron level $(\mu \mathrm{g} / \mathrm{dL})$ and TIBC $(\mu \mathrm{g} / \mathrm{dL})$ were measured with Hitachi Automatic Analyzer 7600 (Hitachi; Japan) using Bathophenanthroline direct method. Iron intake ( $\mathrm{mg} /$ day) was estimated using 24-hour dietary recall questionnaire validated for good reliability and reproducibility in a previous study (17).

Anthropometric variables such as height and weight were measured by trained medical personnel at the mobile examination center. Body mass index (BMI) was calculated as $\mathrm{kg} / \mathrm{m}^{2}$, and was categorized into four groups (underweight, $<18.5 \mathrm{~kg} / \mathrm{m}^{2}$; normal, $18.5-22.9 \mathrm{~kg} / \mathrm{m}^{2} ;$ pre-obese, $23-24.9 \mathrm{~kg} / \mathrm{m}^{2}$; obese, $\geq 25$ $\mathrm{kg} / \mathrm{m}^{2}$ ) based on the Korean Society for the Study of Obesity guideline (18).

\section{Statistical analysis}


Since KNHANES acquired data by multistage, stratified, and probability sampling method to represent the non-institutionalized Korean citizens, all statistical analyses were performed with complex sample analysis method. Moreover, since KCDC assigned sampling weights to account for complex survey design, non-responders, and post-stratification, those sampling weights were applied in all analyses of the current study (14).

The subjects' general characteristics were compared between men and women using Student's t-test for continuous variables with normal distribution, chi-square test for categorical variables, and MannWhitney test for continuous variables without normal distribution (serum ferritin level and daily iron intake). Dose-dependent relationships between the ferritin level divided into tertiles and spirometry results (predicted $\mathrm{FEV}_{1} \%$, predicted $\mathrm{FVC} \%$, and $\mathrm{FEV}_{1} / \mathrm{FVC} \%$ ) were determined using Jonckheere-Terpstra trend test.

Linear regression analysis was used to evaluate the association between serum ferritin level and lung function. Since daily iron intake, hemoglobin, TIBC, and serum iron level can affect ferritin concentration, and previous studies reported limitations due to insufficient information of the variables $(12,13)$, those variables were included in the analysis as covariates. In addition, other variables that affect lung function were included (age, height, residential area, and education). Due to left-sided skewed distribution of the serum ferritin, log-transformed value was applied in the linear regression analysis. Multivariate logistic analysis was used to evaluate the odds ratio (OR) for OLD and RLD. Before statistical analysis, multicollinearity test was performed to identify any inter-associations between the variables.

To account for discrepancy between self-reported smoking status and that through biochemical verification, sensitivity analysis was performed on the basis of measurement of the urinary cotinine levels. In the sub-group analysis, cut-off value of $50-\mathrm{ng} / \mathrm{mL}$ urinary cotinine was determined for active smokers; hence, urinary cotinine of $<50 \mathrm{ng} / \mathrm{mL}$ was used to indicate tobacco-naïve population (19).

All tests were two-tailed, and $P$-value of $<0.05$ were considered as statistically significant. All statistical analyses were performed using IBM Statistical Package for the Social Sciences (SPSS) for Windows v24.0 (IBM Corp., Armonk, NY, USA).

\section{Results}

General characteristics of the study subjects are presented in Table 1. The total cohort of 3,221 subjects was comprised of $342(10.6 \%)$ men and 2,879 (89.3\%) women. Significant differences of age $(P=0.005)$, educational level $(P<0.001)$, height $(P<0.001)$, BMI $(P=0.048)$, iron profiles, and spirometry results were observed between the men and women. Specific characteristics of women according to pre- and postmenopausal status are summarized in Supplementary Table S1.

Dose-dependent relationship between serum ferritin and PFT is presented in Figure 1. Predicted FVC\% was negatively associated with the serum ferritin level of post-menopausal women ( $P$ for trend $=0.044$ ), but not that of pre-menopausal women $(P$ for trend $=0.550)$. $\mathrm{FEV}_{1} / \mathrm{FVC} \%$ was positively associated with 
the serum ferritin level of pre-menopausal women $(P$ for trend $=0.004)$, but not that of post-menopausal women ( $P$ for trend $=0.342$ ). Predicted $\mathrm{FEV}_{1} \%$ was not associated with the serum ferritin of both pre- and post-menopausal women ( $P$ for trend $=0.282$; $P$ for trend $=0.430$, respectively). In men, no significant trend of $\mathrm{FEV}_{1} \%(P$ for trend $=0.927), \mathrm{FVC} \%(P$ for trend $=0.785)$, and $\mathrm{FEV}_{1} / \mathrm{FVC} \%(P$ for trend $=0.293)$ was observed.

The relationship between ferritin and lung function in men and women is shown in Table 2. Model 1 was adjusted for age and height, model 2 was adjusted for additional factors of residence, education, and household income, and model 3 was adjusted for additional factors of hemoglobin, serum iron, TIBC, and daily iron intake. In the crude model and model with adjustment of multivariates, the predicted $\mathrm{FVC} \%$ was inversely correlated to the ferritin level of women, with statistical significance; the predicted $\mathrm{FEV}_{1} \%$ was inversely correlated with that with significance only after adjustment for multivariates; the $\mathrm{FEV}_{1} / \mathrm{FVC} \%$ was significantly associated with that with low-strength and divergent relationship with sequential application of variables. In men, no significant relationship of the parameters was observed.

The relationship between ferritin and lung function in pre- and post-menopausal women is shown in Table 3. The predicted FVC\% was inversely correlated with the serum ferritin level of post-menopausal women, with statistical significance even after adjustment for covariates; whereas, the predicted FVC\% was not significantly associated with that of pre-menopausal women. In both pre- and post-menopausal women, a trend of decrease of the predicted $\mathrm{FEV}_{1} \%$ with increases of the serum ferritin level was observed, with statistical significance for the post-menopausal group only after adjustment for covariates. The $\mathrm{FEV}_{1} / \mathrm{FVC} \%$ was significantly associated with the ferritin level of pre-menopausal women, with lower strength of the association after adjustment for multivariates; whereas, it was not significantly associated with that of post-menopausal women.

The multivariate ORs for RLD and OLD are shown in Table 4. In post-menopausal women, the OR for RLD was increased 2.285-fold at the highest tertile of ferritin level (Q3) versus the lowest tertile (Q1), and 1.560-fold at the middle tertile (Q2) versus Q1; whereas, in pre-menopausal women, the OR for RLD was not statistically associated with the ferritin level. In both pre-menopausal and post-menopausal women, the OR for OLD was not statistically associated with the ferritin level. In men, the ORs of RLD and OLD were not calculated due to no subjects at Q3 level.

ORs for RLD in post-menopausal women classified into two groups of 781 subjects with HRT and 1,356 subjects without HRT is shown in Supplementary Table S2. In subjects with HRT, the OR for RLD was 2.384-fold higher at Q3 and 2.578-fold higher at Q2 than at Q1, respectively ( $P$ trend $=0.021)$; whereas, in subjects without HRT, it was 1.986-fold higher at Q3 and 1.079-fold higher at Q2 than at Q1, respectively $(P$ trend $=0.017)$.

In the sensitivity analysis with urinary cotinine concentration, data of 58 pre-menopausal women and 241 post-menopausal women were obtained. In post-menopause women, the OR for RLD was 1.371-fold 
higher at Q3 and 1.570-fold higher at Q2 than at Q1 ( $P$ trend $=0.564$, Supplementary Table S3). In premenopausal women, the ORs for RLD and OLD were not obtained due to no subjects at Q3 level.

\section{Discussion}

To the best of our knowledge, this is the first study including a nationally representative Korean population to report the association between ferritin level and lung function in the tobacco-naïve population. We observed that post-menopausal women with elevated ferritin level showed an increased risk for RLD, a decrease of the predicted FVC\%, and increased risk for RLD; moreover, in those women, the increase of ferritin level dose-dependently achieved decreases of the predicted FVC\% $(P$ trend $=0.044)$. In contrast, the pre-menopausal women showed no relationship between the ferritin level and predicted value of $\mathrm{FEV}_{1} \%$ and $\mathrm{FVC} \%$, but only weak positive correlation of the ferritin level with the $\mathrm{FEV} \mathrm{F}_{1} / \mathrm{FVC} \%$. Linear regression analysis revealed some trends of increased $\mathrm{FEV}_{1} / \mathrm{FVC} \%$, but with less tendency with sequential application of covariates. In the tobacco-naïve population, the men showed no significant association between the ferritin level and lung function.

Previous studies on the association between ferritin and lung function reported conflicting results $(12,13$, 20). Ghio and colleagues reported that ferritin was associated with airway obstruction in non-smokers $(r=$ $-0.155, P<0.001)$; however, those authors did not use multivariate analysis or include serum iron, TIBC, sex, and age as independent variables (20). In our study, correlation analysis revealed that the highest ferritin level was associated with the lowest FEV1/FVC\% in non-smokers $(r=-0.135, P<0.001)$, but the significance of association was lost with multivariate application. Brigham and colleagues reported that higher ferritin level was associated with lower prevalence of asthma in women; however, those authors did not include the variables of inflammatory status that can affect ferritin level, daily iron intake, or menopause status (12). Lee and colleagues conducted a study including KNHANES data to evaluate the relationship between ferritin and lung function, but they included only subjects with smoking, and did not analyze the other iron indices such as serum iron level, TIBC, and transferrin. To evaluate the relationship between ferritin and lung function, studies focused on various influencing factors of ferritin level or lung function are needed. Based on these facts, in the current study, we conducted multivariate analysis including iron indices as potential confounding variables, excluded subjects with inflammatory status, and performed sub-group analyses according to the menopausal status and HRT use.

In previous analyses, since categorization of non-smokers according to WHO classification system does not consider second-hand smoking, a few passive smokers may be included in the non-smoking group. To overcome this limitation, we additionally used the questionnaire item on exposure to second-hand smoke at home and in the workplace, in order to distinguish the tobacco-naïve population. However, since self-reporting questionnaire is an unreliable tool to assess the smoking status, since it underestimates the smoking rates, especially among Korean women (21), to verify the tobacco-naïve population, we used urinary cotinine concentration of $<50 \mathrm{ng} / \mathrm{mL}$ as objective index (19). KNHANES assessed the subject's urinary cotinine concentration only in 2010 and 2011, and data of 58 pre-menopausal women and 241 post-menopausal women with never smoking without second-hand smoking were available. In our study, 
linear regression model revealed negative association between the ferritin level and predicted FVC\% in post-menopausal women, without statistical significance $(\mathrm{B}=-6.319,95 \% \mathrm{Cl}:-17.064-4.425, P=0.245)$; in addition, logistic regression analysis revealed no statistical significant relationship among those parameters.

These results can be explained by several phenomena. First, ferritin is related to oxidative stress: High iron storage increases ferritin concentration and promotes oxidative stress, which induces several inflammatory responses and tissue injury, and consequently, even cell death (4), and iron accumulation in the alveolar macrophage increases tumor necrosis factor (TNF) expression which results in interstitial lung injuries (22). Second, ferritin plays a protective role against tissue damage caused by oxygen free radicals: Oxidative stress of the tissues induces synthesis of ferritin, the major mammalian iron-storage protein, through catalysis of Fe (II) oxidation to $\mathrm{Fe}$ (III), and this detoxification process protects the tissues from oxidative damage (23). One study on ferritin synthesis in the liver slice model reported a 6-fold increase of those levels for rats treated with phorone, a glutathione-depleting drug that amplifies reactive oxygen species (ROS) production (3). With regard to applicability of that result, elevated ferritin that reflects persistent inflammatory reactions can cause structural damage of the pulmonary system. A study including patients with thalassemia major reported that serum ferritin was associated with restrictive pulmonary dysfunction and poor prognosis (24). Although the mechanism of RLD in these patients is unclear, it could be explained in part by stepwise process of iron accumulation in the lung, ferritin production, generation of hydroxyl free radicals, and fibrotic injury related to TGF- $\beta$, in order $(25,26)$. These clinical findings are supported by an in vivo animal study which showed that ferroportin gene mutation in the mouse resulted in disrupted pulmonary iron regulatory system, alveolar iron accumulation, oxidative stress with elevation of heme-oxygenase-1, induction of lipid peroxidation, and pulmonary restriction (27).

We observed that ferritin was associated with reduction of lung function only in post-menopausal women, which could be explained by the fact that estrogen is essential for maintenance and regulation of the female reproductive system (28), and is used in HRT at post-menopause due to its various positive effects. Previous reports indicate that estrogen has a protective role in lung function. Pata and colleagues reported that HRT in post-menopausal women improved the lung function, and regardless of the type of therapy, achieved significant improvement of the $\mathrm{FVC} \%$ and $\mathrm{FEV}_{1} \%$ after 3 months (29). In this study, we performed sensitivity analysis to evaluate whether HRT was a contributing factor of the RLD prevalence in post-menopausal women. Among the post-menopausal women included, 781 (36.5\%) received HRT and 1,356 (63.5\%) did not receive HRT (Supplementary Table S1). Logistic regression analysis revealed a trend of increasing RLD in subjects without HRT, and high OR for RLD due to high serum ferritin level in post-menopausal women.

We observed significant association between the ferritin level and lung function only in the postmenopausal group, despite the finding of higher mean level of ferritin in men versus post-menopausal women, which could be explained by the differences of sex hormone signaling between the sexes (30). Patients with Klinefelter's syndrome with absence of testosterone showed a high prevalence of RLD (31), 
and in a cross-sectional study, elderly men showed inverse relationship of the testosterone level and inflammatory marker, which suggests that testosterone is a potential contributing factor of chronic inflammatory disease (32). To identify underlying mechanism(s) of sex differences, biological study at the hormonal level is needed.

Our study has several limitations. First, because KNHANES is a cross-sectional study, temporal aspects are not considered, and investigators should cautiously interpret the causal relationship between ferritin and lung function in tobacco-naïve post-menopausal women. Second, since self-reporting survey tends to underestimate the smoking rate (21), the size of tobacco-naïve population may be overestimated; in addition, definition of tobacco-naïve population was based on questionnaire survey, and the subject's responses may not meet all criteria of the definition. Verification of the definition by urine cotinine concentration was attempted, but limited data of 58 pre-menopausal women and 241 post-menopausal women were available.

Within these limitations, this is the first study including a nationally representative Korean population to report the associations of lung function and ferritin in the tobacco-naïve population according to iron indices, systemic condition, socio-economic status, second-hand smoking, menopausal status, and HRT.

\section{Abbreviations}

KCDC; Korean Center for Disease Control and Prevention, KNHANES; Korea National Health and Nutritional Examination Survey, TIBC; Total iron binding capacity, PFT; Pulmonary function test, IRB; Institutional Review Board, OLD; Obstructive lung disease, RLD; Restrictive lung disease, $\mathrm{FEV}_{1}$; Forced expiratory volume in 1 second, FVC; Forced vital capacity, HRT; Hormonal therapy, BMI; Body mass index, OR; odds ratio, SPSS; Statistical Package for the Social Sciences, Q3; Highest tertile, Q2; middle tertile, Q1; Lowest tertile, TNF; Tumor necrosis factor, ROS; Reactive oxygen species.

\section{Conclusions}

In tobacco-naïve post-menopausal women, high serum ferritin level was significantly associated with decreased value of predicted FVC\%. Moreover, high serum ferritin level was associated with increased risk of restrictive lung disease, which may be related to the oxidative stress induced by ferritin or persistent inflammatory reaction represented by elevated ferritin concentration. Further studies are needed to identify underlying mechanisms of these relationships.

\section{Declarations}

\section{Conflict of interest}

The authors declare no conflicts of interests. 


\section{Funding}

This study was supported in part by Korean National Research Foundation grants 2019M3E5D1A02070863.

\section{Authors' contributions}

$\mathrm{TY}, \mathrm{HJ}$, and $\mathrm{JH}$ contributed to the study design, data analysis, data interpretation, and reviewing and writing of this manuscript. TY and $\mathrm{HJ}$ contributed equally to this article. All authors read and approved the final manuscript.

\section{References}

1. Munro HN, Linder MC. Ferritin: structure, biosynthesis, and role in iron metabolism. Physiol Rev. 1978;58(2):317-96.

2. Arosio P, Levi S. Ferritin, iron homeostasis, and oxidative damage. Free Radic Biol Med. 2002;33(4):457-63.

3. Cairo G, Tacchini L, Pogliaghi G, Anzon E, Tomasi A, Bernelli-Zazzera A. Induction of ferritin synthesis by oxidative stress transcriptional and post-transcriptional regulation by expansion of the "free" iron pool. J Biol Chem. 1995;270(2):700-3.

4. Emerit J, Beaumont C, Trivin F. Iron metabolism, free radicals, and oxidative injury. Biomed Pharmacother. 2001;55(6):333-9.

5. Jurado RL. Iron, infections, and anemia of inflammation. Clin Infect Dis. 1997;25(4):888-95.

6. Konijn A, Hershko C. Ferritin synthesis in inflammation: I. Pathogenesis of impaired iron release. $\mathrm{Br} \mathrm{J}$ Haematol. 1977;37(1):7-16.

7. Donato F, Pasini G, Buizza M, Fantoni C, Tomasi E, Tani M, et al. Tobacco smoking, occupational exposure and chronic respiratory disease in an Italian industrial area. Monaldi archives for chest disease $=$ Archivio Monaldi per le malattie del torace. 2000;55(3):194-200.

8. Kamp DW, Srinivasan M, Weitzman SA. Cigarette smoke and asbestos activate poly-ADP-ribose polymerase in alveolar epithelial cells. J Investig Med. 2001;49(1):68-76.

9. Kim J, Wessling-Resnick M. The role of iron metabolism in lung inflammation and injury. Journal of allergy \& therapy. 2012;3(Suppl 4).

10. Philippot Q, Deslée G, Adair-Kirk TL, Woods JC, Byers D, Conradi S, et al. Increased iron sequestration in alveolar macrophages in chronic obtructive pulmonary disease. PLoS One. 2014;9(5):e96285.

11. Nelson ME, O'Brien-Ladner AR, Wesselius LJ. Regional variation in iron and iron-binding proteins within the lungs of smokers. Am J Respir Crit Care Med. 1996;153(4):1353-8.

12. Brigham EP, McCormack MC, Takemoto CM, Matsui EC. Iron status is associated with asthma and lung function in US women. PLoS One. 2015;10(2):e0117545. 
13. Lee CH, Goag EK, Lee SH, Chung KS, Jung JY, Park MS, et al. Association of serum ferritin levels with smoking and lung function in the Korean adult population: analysis of the fourth and fifth Korean National Health and Nutrition Examination Survey. Int J Chron Obstruct Pulmon Dis. 2016;11:3001.

14. Kweon S, Kim Y, Jang M-j, Kim Y, Kim K, Choi S, et al. Data resource profile: the Korea national health and nutrition examination survey (KNHANES). Int J Epidemiol. 2014;43(1):69-77.

15. Miller MR, Hankinson J, Brusasco V, Burgos F, Casaburi R, Coates A, et al. Standardisation of spirometry. Eur Respir J. 2005;26(2):319-38.

16. Vogelmeier CF, Criner GJ, Martinez FJ, Anzueto A, Barnes PJ, Bourbeau J, et al. Global strategy for the diagnosis, management, and prevention of chronic obstructive lung disease 2017 report. GOLD executive summary. Am J Respir Crit Care Med. 2017;195(5):557-82.

17. Carter RL, Sharbaugh CO, Stapell CA. Reliability and validity of the 24-hour recall. J Am Diet Assoc. 1981;79(5):542-7.

18. Seo MH, Lee W-Y, Kim SS, Kang J-H, Kang J-H, Kim KK, et al. 2018 Korean Society for the Study of Obesity Guideline for the Management of Obesity in Korea. Journal of obesity \& metabolic syndrome. 2019;28(1):40.

19. Moyer TP, Charlson JR, Enger RJ, Dale LC, Ebbert JO, Schroeder DR, et al. Simultaneous analysis of nicotine, nicotine metabolites, and tobacco alkaloids in serum or urine by tandem mass spectrometry, with clinically relevant metabolic profiles. Clin Chem. 2002;48(9):1460-71.

20. Ghio AJ, Hilborn ED. Indices of iron homeostasis correlate with airway obstruction in an NHANES III cohort. Int J Chron Obstruct Pulmon Dis. 2017;12:2075.

21. Jung-Choi K-H, Khang $\mathrm{Y}-\mathrm{H}, \mathrm{Cho} \mathrm{H}-\mathrm{J}$. Hidden female smokers in Asia: a comparison of self-reported with cotinine-verified smoking prevalence rates in representative national data from an Asian population. Tob Control. 2012;21(6):536-42.

22. Wesselius LJ, Smirnov IM, Nelson ME, O'Brien-Ladner AR, Flowers CH, Skikne BS. Alveolar macrophages accumulate iron and ferritin after in vivo exposure to iron or tungsten dusts. $\mathrm{J}$ Lab Clin Med. 1996;127(4):401-9.

23. Wang Z, Li C, Ellenburg M, Soistman E, Ruble J, Wright B, et al. Structure of human ferritin L chain. Acta Crystallographica Section D: Biological Crystallography. 2006;62(7):800-6.

24. Bourli E, Dimitriadou M, Economou M, Vlachaki E, Christoforidis A, Maratou E, et al. Restrictive pulmonary dysfunction and its predictors in young patients with $\beta$-thalassaemia major. Pediatr Pulmonol. 2012;47(8):801-7.

25. Factor JM, Pottipati SR, Rappoport I, Rosner IK, Lesser ML, Giardina PJ. Pulmonary function abnormalities in thalassemia major and the role of iron overload. Am J Respir Crit Care Med. 1994;149(6):1570-4.

26. Sritippayawan S, Lekhanont P, Harnruthakorn C, Samransamruajkit R, Deerojanawong J, Seksarn P, et al. Restrictive lung disease and serum TGF-[beta] 1 in thalassemia major children. Asian Pac $\mathrm{J}$ Allergy Immunol. 2005;23(2-3):121. 
27. Neves J, Leitz D, Kraut S, Brandenberger C, Agrawal R, Weissmann N, et al. Disruption of the hepcidin/ferroportin regulatory system causes pulmonary iron overload and restrictive lung disease. EBioMedicine. 2017;20:230-9.

28. Zhu BT, Conney AH. Functional role of estrogen metabolism in target cells: review and perspectives. Carcinogenesis. 1998;19(1):1-27.

29. Pata Ö, Atiş S, Öz AU, Yazici G, Tok E, Pata C, et al. The effects of hormone replacement therapy type on pulmonary functions in postmenopausal women. Maturitas. 2003;46(3):213-8.

30. Sathish V, Martin YN, Prakash Y. Sex steroid signaling: implications for lung diseases. Pharmacol Ther. 2015;150:94-108.

31. Morales P, Furest I, Marco V, Macian V, Moreno B, Jimenez-Cruz JF. Pathogenesis of the lung in restrictive defects of Klinefelter's syndrome. Chest. 1992;102(5):1550-2.

32. Maggio M, Basaria S, Ble A, Lauretani F, Bandinelli S, Ceda GP, et al. Correlation between testosterone and the inflammatory marker soluble interleukin-6 receptor in older men. The Journal of Clinical Endocrinology \& Metabolism. 2006;91(1):345-7.

\section{Tables}

Table 1. General characteristics of the study subjects 


\begin{tabular}{|c|c|c|c|c|}
\hline & Total & Men & Women & $P$-value \\
\hline Number (\%) & 3,221 & $342(10.6)$ & $2,879(89.3)$ & \\
\hline Population size (\%) & $21,264,098$ & $2,436,715$ (11.5) & $18,827,383(88.5)$ & \\
\hline Age, mean (SE) & $58.7(0.2)$ & $60.25(0.6)$ & $58.5(0.2)$ & 0.005 \\
\hline Residential area, \% (SE) & & & & 0.388 \\
\hline Urban & $75.3(2.1)$ & $73.2(3.5)$ & $75.6(2.1)$ & \\
\hline Rural & $24.7(2.1)$ & $26.8(3.5)$ & $24.4(2.1)$ & \\
\hline Education, \% (SE) & & & & $<0.001$ \\
\hline Middle school or lower & $52.8(1.4)$ & $33.4(3.2)$ & $55.3(1.4)$ & \\
\hline High school & $27.8(1.1)$ & $27.0(2.8)$ & $27.9(1.2)$ & \\
\hline College or more & $19.4(1.1)$ & $39.6(3.3)$ & $16.8(1.1)$ & \\
\hline Height, $\mathrm{cm}$, mean (SE) & $156.4(0.1)$ & $167.3(0.3)$ & $155.0(5.7)$ & $<0.001$ \\
\hline Body mass index, \% (SE) & & & & 0.048 \\
\hline Underweight & $1.7(0.4)$ & $1.4(1.0)$ & $1.7(0.3)$ & \\
\hline Normal & $35.7(1.1)$ & $29.7(2.9)$ & $36.5(1.2)$ & \\
\hline Pre-obese & $24.9(1.0)$ & $32.4(3.0)$ & $23.9(1.0)$ & \\
\hline Obese & $37.7(1.2)$ & $36.4(3.1)$ & $37.8(1.2)$ & \\
\hline Household Income, \% (SE) & & & & 0.658 \\
\hline Lowest & $27.4(1.2)$ & $26.6(3.1)$ & $27.6(1.3)$ & \\
\hline Lower middle & $26.6(1.1)$ & $28.2(3.0)$ & $26.4(1.1)$ & \\
\hline Higher middle & $21.0(1.0)$ & $18.5(2.4)$ & $21.4(1.0)$ & \\
\hline Highest & $24.9(1.1)$ & $26.7(2.7)$ & $24.7(1.1)$ & \\
\hline \multicolumn{5}{|l|}{ Iron profile } \\
\hline Hemoglobin, g/dL, mean (SE) & $13.3(0.0)$ & $15.0(0.1)$ & $13.1(1.1)$ & $<0.001$ \\
\hline TIBC, ug/dL, mean (SE) & $316.6(0.8)$ & $307.9(2.3)$ & $317.6(0.8)$ & $<0.001$ \\
\hline Serum iron, ug/dL, mean (SE) & $104.4(0.7)$ & $124.8(2.2)$ & $101.9(0.7)$ & $<0.001$ \\
\hline Median value of serum ferritin, $\mathrm{ng} / \mathrm{mL}$ & 51.5 & 87.3 & 48.3 & $<0.001$ \\
\hline IQR & $28.5-85.6$ & $56.8-140.6$ & $26.6-78.9$ & \\
\hline Median value of iron intake, mg/day & 12.1 & 15.1 & 11.8 & $<0.001$ \\
\hline IQR & $8.3-17.8$ & $10.6-21.8$ & $8.1-17.3$ & \\
\hline FVC, Liter & & & & $<0.001$ \\
\hline Mean (SE) & $3.0(0.0)$ & $4.0(0.0)$ & $2.9(0.0)$ & \\
\hline Median (IQR) & $2.9(2.6-3.4)$ & $3.9(3.5-4.5)$ & $2.9(2.5-3.2)$ & \\
\hline Predicted FVC\%, mean (SE) & $93.6(0.2)$ & $90.7(0.6)$ & $93.9(0.2)$ & $<0.001$ \\
\hline $\mathrm{FEV}_{1}$, Liter & & & & $<0.001$ \\
\hline Mean (SE) & $2.4(0.0)$ & $3.1(0.0)$ & $2.3(0.0)$ & \\
\hline Median (IQR) & $2.3(2.0-2.7)$ & $3.1(2.7-3.5)$ & $2.3(1.9-2.6)$ & \\
\hline predicted $\mathrm{FEV}_{1} \%$, mean (SE) & $94.9(0.2)$ & $92.9(0.7)$ & $95.1(0.2)$ & 0.005 \\
\hline $\mathrm{FEV}_{1} / \mathrm{FVC} \%$, mean (SE) & $78.9(0.0)$ & $76.1(0.0)$ & $79.3(0.1)$ & $<0.001$ \\
\hline
\end{tabular}

Data are presented as weighted mean (SE) or weighted percentage (SE), unless otherwise stated. 

second; TIBC, total iron binding capacity.

Table 2. Relationship between ferritin level and lung function according to sex.

\begin{tabular}{|c|c|c|c|c|}
\hline Predicted $\mathrm{FEV}_{1} \%$ & & B & $95 \% \mathrm{CI}$ & $P$-value \\
\hline \multirow[t]{4}{*}{ Men $(\mathrm{n}=342)$} & Crude & -1.029 & $-7.161,5.102$ & 0.741 \\
\hline & Model 1 & -0.093 & $-5.926,5.740$ & 0.975 \\
\hline & Model 2 & 1.072 & $-4.648,6.791$ & 0.712 \\
\hline & Model 3 & -1.113 & $-7.012,4.785$ & 0.710 \\
\hline \multirow[t]{4}{*}{ Women $(\mathrm{n}=2,879)$} & Crude & 0.521 & $-0.953,1.994$ & 0.488 \\
\hline & Model 1 & -1.423 & $-2.972,0.126$ & 0.072 \\
\hline & Model 2 & -1.468 & $-3.035,0.099$ & 0.066 \\
\hline & Model 3 & -2.808 & $-5.475,-0.141$ & 0.039 \\
\hline \multicolumn{5}{|l|}{ Predicted FVC\% } \\
\hline \multirow[t]{4}{*}{ Men $(n=342)$} & Crude & 0.134 & $-4.900,5.259$ & 0.959 \\
\hline & Model 1 & -1.245 & $-5.913,3.424$ & 0.600 \\
\hline & Model 2 & -0.596 & $-5.300,4.108$ & 0.803 \\
\hline & Model 3 & -2.399 & $-7.822,3.025$ & 0.384 \\
\hline \multirow[t]{4}{*}{ Women $(\mathrm{n}=2,879)$} & Crude & -2.559 & $-3.883,-1.235$ & $<0.001$ \\
\hline & Model 1 & -1.783 & $-3.213,-0.353$ & 0.015 \\
\hline & Model 2 & -1.797 & $-3.244,-0.350$ & 0.015 \\
\hline & Model 3 & -4.111 & $-5.924,-2.298$ & $<0.001$ \\
\hline \multicolumn{5}{|l|}{$\mathrm{FEV}_{1} / \mathrm{FVC} \%$} \\
\hline \multirow[t]{4}{*}{ Men $(n=342)$} & Crude & 0.020 & $-0.010,0.050$ & 0.190 \\
\hline & Model 1 & 0.009 & $-0.019,0.037$ & 0.533 \\
\hline & Model 2 & 0.011 & $-0.018,0.039$ & 0.468 \\
\hline & Model 3 & 0.006 & $-0.024,0.036$ & 0.704 \\
\hline \multirow[t]{4}{*}{ Women $(\mathrm{n}=2,879)$} & Crude & -0.017 & $-0.023,-0.012$ & $<0.001$ \\
\hline & Model 1 & 0.006 & $-0.0003,0.011$ & 0.067 \\
\hline & Model 2 & 0.005 & $-0.001,0.011$ & 0.093 \\
\hline & Model 3 & 0.009 & $0.001,0.018$ & 0.032 \\
\hline
\end{tabular}

Model 1 is adjusted for age and height.

Model 2 is adjusted for age, height, residence, education, and household income.

Model 3 is adjusted for age, height, residence, education, household income, hemoglobin, serum iron, daily iron intake, and TIBC. 
Table 3. Relationship between ferritin level and lung function according to menopause status.

\begin{tabular}{|c|c|c|c|c|}
\hline Predicted FEV $1 \%$ & & B & $95 \% \mathrm{CI}$ & $P$-value \\
\hline \multirow[t]{4}{*}{ Pre-menopause $(n=742)$} & Crude & 0.978 & $-1.170,3.126$ & 0.371 \\
\hline & Model 1 & 1.044 & $-1.106,3.194$ & 0.340 \\
\hline & Model 2 & 0.928 & $-1.193,3.049$ & 0.390 \\
\hline & Model 3 & -1.761 & $-5.194,1.672$ & 0.314 \\
\hline \multirow[t]{4}{*}{ Post-menopause $(\mathrm{n}=2,137)$} & Crude & -1.402 & $-3.918,1.114$ & 0.274 \\
\hline & Model 1 & -2.013 & $-4.334,0.307$ & 0.089 \\
\hline & Model 2 & -2.714 & $-4.489,0.140$ & 0.066 \\
\hline & Model 3 & -2.847 & $-5.518,-0.177$ & 0.037 \\
\hline \multicolumn{5}{|l|}{ Predicted FVC\% } \\
\hline \multirow[t]{4}{*}{ Pre-menopause $(n=742)$} & Crude & -0.675 & $-2.690,1.339$ & 0.510 \\
\hline & Model 1 & -0.523 & $-2.541,1.496$ & 0.611 \\
\hline & Model 2 & -0.551 & $-2.528,1.426$ & 0.584 \\
\hline & Model 3 & -0.501 & $-2.541,1.539$ & 0.630 \\
\hline \multirow[t]{4}{*}{ Post-menopause $(n=2,137)$} & Crude & -2.689 & $-4.777,-0.600$ & 0.012 \\
\hline & Model 1 & -2.672 & $-4.741,-0.602$ & 0.012 \\
\hline & Model 2 & -2.761 & $-4.836,-0.686$ & 0.009 \\
\hline & Model 3 & -4.148 & $-6.556,-1.739$ & 0.001 \\
\hline \multicolumn{5}{|l|}{$\mathrm{FEV}_{1} / \mathrm{FVC} \%$} \\
\hline \multirow[t]{4}{*}{ Pre-menopause $(\mathrm{n}=742)$} & Crude & 0.010 & $0.001,0.019$ & 0.036 \\
\hline & Model 1 & 0.010 & $0.001,0.020$ & 0.037 \\
\hline & Model 2 & 0.010 & $0.00021,0.019$ & 0.045 \\
\hline & Model 3 & 0.013 & $-0.001,0.028$ & 0.073 \\
\hline \multirow[t]{4}{*}{ Post-menopause $(\mathrm{n}=2,137)$} & Crude & 0.0005 & $-0.009,0.010$ & 0.920 \\
\hline & Model 1 & 0.003 & $-0.005,0.012$ & 0.473 \\
\hline & Model 2 & 0.003 & $-0.006,0.011$ & 0.566 \\
\hline & Model 3 & 0.008 & $-0.003,0.019$ & 0.141 \\
\hline
\end{tabular}

Model 1 is adjusted for age and height.

Model 2 is adjusted for age, height, residence, education, and household income.

Model 3 is adjusted for age, height, residence, education, household income, hemoglobin, serum iron, daily iron intake, and TIBC. 
Table 4. Risk of restrictive lung disease (RLD) and obstructive lung disease (OLD) according to menopause status (742 in pre- and 2,137 in post-menopausal women).

\begin{tabular}{|l|c|c|l|c|c|}
\hline & \multicolumn{2}{|c|}{ OLD } & \multicolumn{3}{c|}{ RLD } \\
\hline & OR (95\% CI) & P trend & & OR (95\% CI) & $P$ trend \\
\hline Pre-menopause & & 0.751 & Pre-menopause & & 0.999 \\
\hline Q1 & reference & & Q1 & reference & \\
\hline Q2 & $0.656(0.049,8.717)$ & & Q2 & $1.096(0.364,3.302)$ & \\
\hline Q3 & $1.147(0.079,16.635)$ & & Q3 & $1.030(0.248,4.279)$ & \\
\hline Post-menopause & & 0.555 & Post-menopause & & $\mathbf{0 . 0 0 1}$ \\
\hline Q1 & reference & & Q1 & reference & \\
\hline Q2 & $0.824(0.473,1.435)$ & & Q2 & $1.560(0.967,2.517)$ & \\
\hline Q3 & $0.860(0.511,1.448)$ & & Q3 & $2.285(1.424,3.668)$ & \\
\hline
\end{tabular}

OR, odds ratio; CI, confidence interval; OLD, obstructive lung disease; RLD, restrictive lung disease.

\section{Figures}
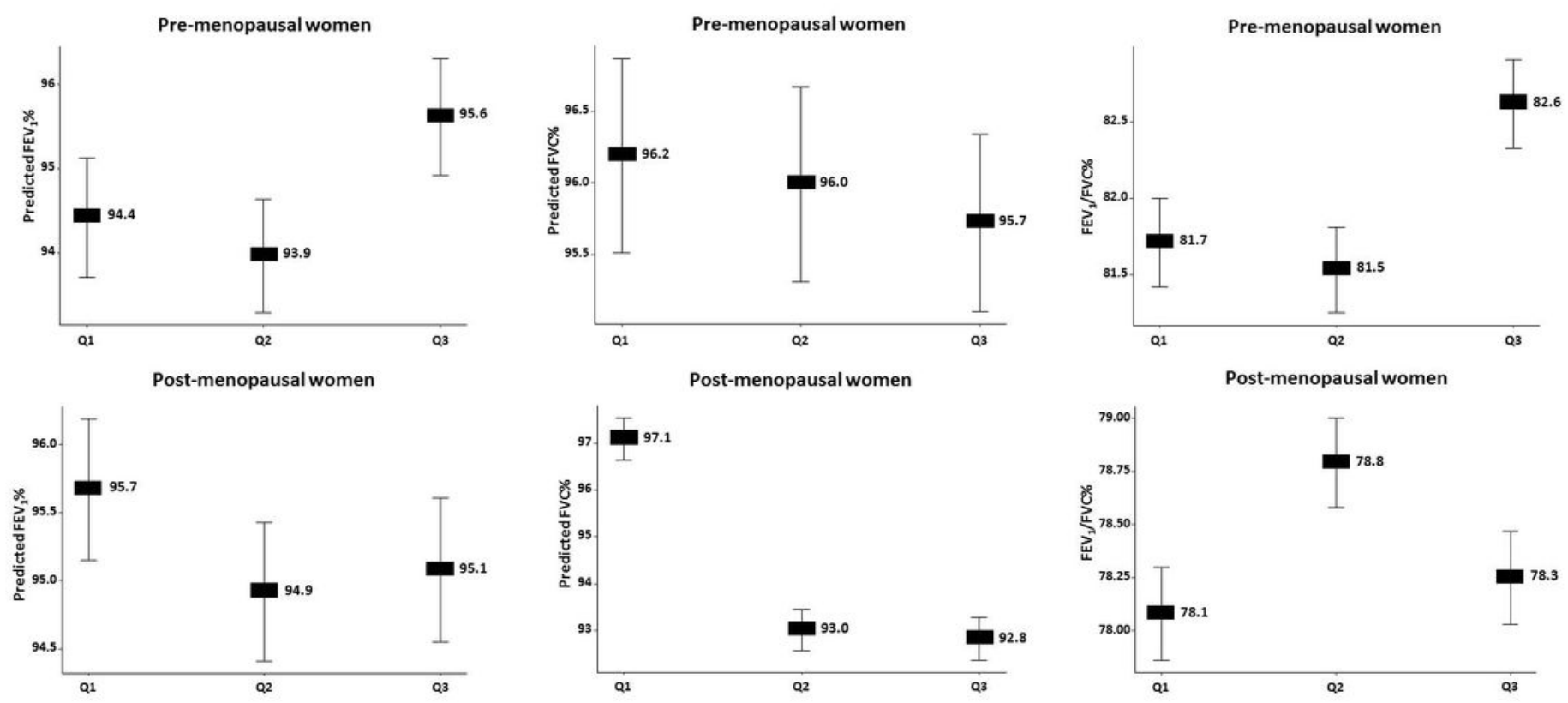

Figure 1 
Dose-dependent relationship between serum ferritin level and measurement values of pulmonary function test in pre- and post-menopausal women.

\section{Supplementary Files}

This is a list of supplementary files associated with this preprint. Click to download.

- SupplementaryTables1028.docx 